\title{
Electron Dynamics in the Diffusion Region of an Asymmetric Magnetic Reconnection
}

\author{
D. B. Graham, ${ }^{1}$ Yu. V. Khotyaintsev, ${ }^{1}$ A. Vaivads, ${ }^{1}$ M. André, ${ }^{1}$ and A. N. Fazakerley ${ }^{2}$ \\ ${ }^{1}$ Swedish Institute of Space Physics, Uppsala, P. O. Box 537, 75121 Uppsala, Sweden \\ ${ }^{2}$ Mullard Space Science Laboratory, University College London, Holmbury St. Mary, Dorking RH5 6NT, United Kingdom
}

(Received 11 December 2013; published 28 May 2014)

\begin{abstract}
During a magnetopause crossing near the subsolar point Cluster observes the ion diffusion region of antiparallel magnetic reconnection. The reconnecting plasmas are asymmetric, differing in magnetic field strength, density, and temperature. Spatial changes in the electron distributions in the diffusion region are resolved and investigated in detail. Heating of magnetosheath electrons parallel to the magnetic field is observed. This heating is shown to be consistent with trapping of magnetosheath electrons by parallel electric fields.
\end{abstract}

DOI: 10.1103/PhysRevLett.112.215004

PACS numbers: 52.35.Vd, 94.05.Pt, 94.30.ch

Magnetic reconnection is the process by which two plasmas with different magnetic fields interact, changing the magnetic field topology, and transferring energy from the magnetic field to particles, resulting in electron and ion acceleration and heating [1]. Symmetric reconnection, in which the two reconnection plasmas have the same properties, has been studied extensively and is relatively well understood [2], and is applicable to Earth's magnetotail. On the other hand, asymmetric reconnection, in which the plasma properties differ, is more general and is relevant to Earth's magnetopause (MP) but is less well understood. A number of numerical studies have shown that asymmetric reconnection is qualitatively different from symmetric reconnection [3-6].

Plasmas reconnect in small regions where the ions and then electrons decouple from the magnetic field, called the ion and electron diffusion regions, respectively. One of the characteristic features of the ion diffusion region (hereafter termed diffusion region) is electron heating parallel to the local magnetic field B. This electron heating has been reported in Earth's magnetotail [7,8] and MP [9], and has been observed in simulations of both symmetric and asymmetric reconnection $[7,8,10]$. For symmetric configurations electron trapping and acceleration due to parallel electric fields $\mathbf{E}_{\|}$is argued to be responsible for the observed electron heating [8]. For asymmetric reconnection electron trapping may also be relevant $[10,11]$, but instabilities such as the lower hybrid drift instability (LHDI) may also be responsible for the heating [9,12]. Indeed, recent simulations and MP observations show that intense electric fields consistent with lower hybrid (LH) waves are produced at the density gradient between the reconnecting plasmas [13-16], but tend to be localized away from the $X$ line along the magnetosphere (MS) separatrices [15]. Determining which processes are responsible for the electron heating in the diffusion region is of intrinsic interest and is crucial for understanding how asymmetric reconnection operates. The purpose of this letter is to examine the electron distributions observed in the diffusion region of asymmetric reconnection at the MP and determine which processes are responsible for the observed heating.

We analyze a MP crossing within 5 Earth radii of the subsolar point observed on 22 April 2008 between 18:03 and 18:13 UT. We use data from Cluster 3 and Cluster 4 (C3 and C4), which are separated by $\sim 30 \mathrm{~km}$, much less than the MS ion skin depth $d_{\mathrm{MS}} \sim 300 \mathrm{~km}$. We use magnetic field data from FGM and STAFF, electric field data from EFW, ion data from CIS-HIA, and electron data from the PEACE instruments [17]. The instruments were all operating in burst mode, with higher resolution data. We use the $I m n$ coordinate system, where $\mathbf{n}$ is the unit vector normal to the assumed MP given by the Shue et al. model [18], $\mathbf{m}=\mathbf{B}_{\mathrm{MS}} \times \mathbf{n} /\left|\mathbf{B}_{\mathrm{MS}}\right|$, where $\mathbf{B}_{\mathrm{MS}}$ is the unperturbed MS $\mathbf{B}$, and $\mathbf{l}=\mathbf{n} \times \mathbf{m}$ completes the system. The guide-field direction is in the $m$ direction and the $n$ direction is closely aligned with the geocentric solar magnetospheric $x$ direction.

This crossing shows large-scale evidence for asymmetric magnetic reconnection. Figures 1(a)-1(c) show the magnetic field $\mathbf{B}$, ion velocity $\mathbf{V}$, and the electron differential energy flux, respectively. The spacecraft begin in the MS, characterized by low-density high-temperature electrons with thermal energy $E \gtrsim 1 \mathrm{keV}$. At 18:04:30 UT the spacecraft enter a northward reconnection outflow jet. The maximum observed outflow speed is $V \sim 250 \mathrm{~km} \mathrm{~s}^{-1}$, in excellent agreement with the predicted $V \sim 280 \mathrm{~km} \mathrm{~s}^{-1}$ from the scalings of Ref. [19]. The outflow consists of both MS electrons and low-temperature magnetosheath (SH) electrons. Between 18:07 and 18:09 UT the spacecraft observe an ion flow reversal. At this time $B_{l}$ reverses direction as the spacecraft cross the MP. By inspection of Fig. 1(a), reconnection is nearly antiparallel. After the south ion outflow the spacecraft enter the $\mathrm{SH}$, where no MS electrons are observed. The spacecraft then partially cross another northward ion outflow either associated with the same or another $X$ line. The blue shaded region in Fig. 1 is the ion diffusion region (justified in more detail below), 

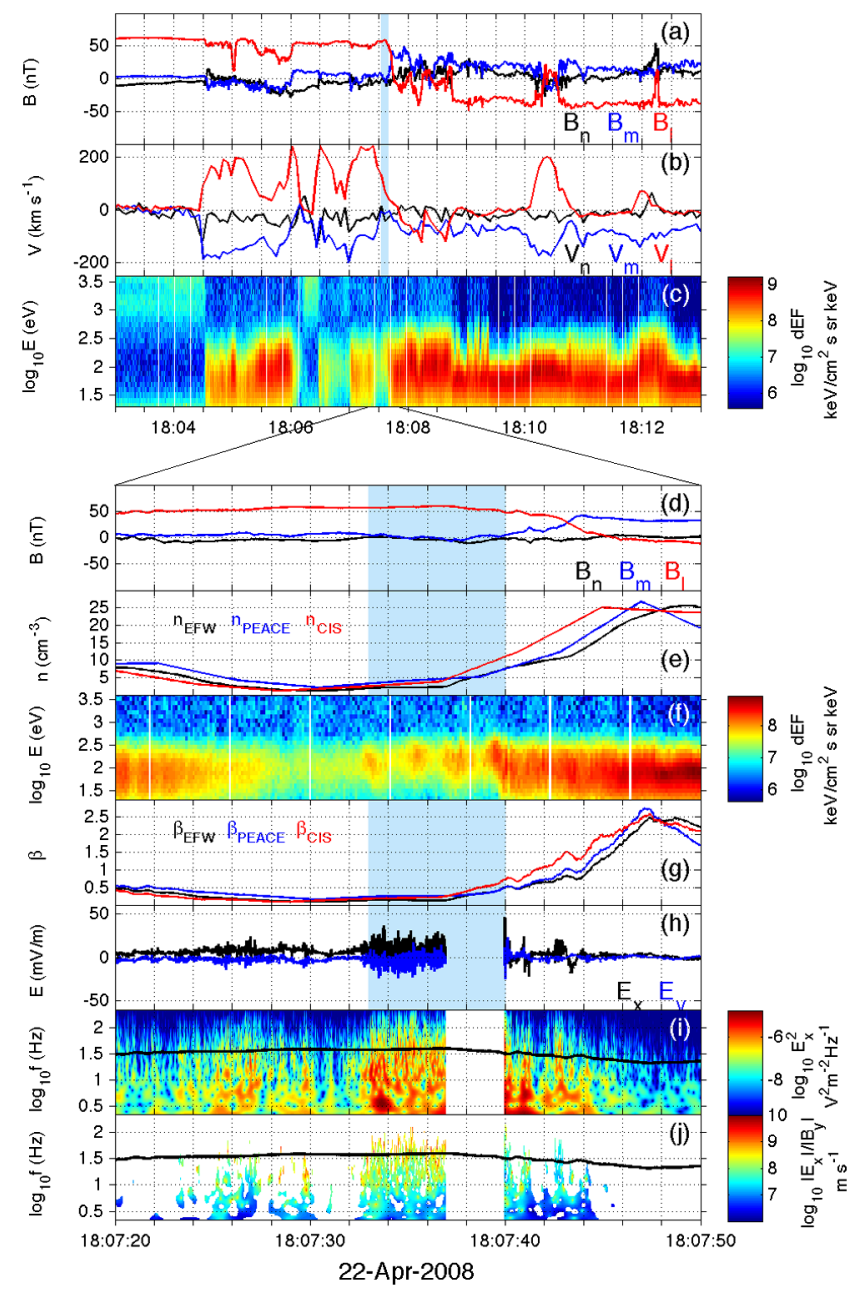

FIG. 1 (color online). The $X$-line crossing observed by $\mathrm{C} 3$ and C4. Panels (a)-(c) show the MP crossing. Panels (d)-(i) show data near the diffusion region. (a) $\mathbf{B}$ in $l m n$ coordinates. (b) $\mathbf{V}$ in Imn coordinates. (c) Omnidirectional electron differential flux. (d) $\mathbf{B}$ in $l m n$ coordinates. (e) $n$. (f) Omnidirectional electron differential energy flux. (g) $\beta$. (h) Electric field in spacecraft coordinates. (i) Wavelet transform of $E_{x}$. (j) $\left|E_{x}\right| /\left|B_{y}\right|$. The black line in panels (i) and (j) is the local lower hybrid frequency. The blue shading indicates the diffusion region.

which is observed between the outflow reversal, close to the stagnation point, and is on the MS side of the $X$ line.

We now investigate the plasma conditions in the ion diffusion region in detail. Figure 1(d) shows that $\mathbf{B} \approx \mathbf{B}_{\mathrm{MS}}$ in the diffusion region, meaning the spacecraft have not yet crossed the MP. Solar wind data from ACE imply that the SH magnetic field is southward and stable throughout the interval. The density $n \sim 5 \mathrm{~cm}^{-3}$ of the diffusion region, shown in Fig. 1(e), is approximately an order of magnitude above the unperturbed MS density $n_{\mathrm{MS}} \sim 0.5 \mathrm{~cm}^{-3}$ due to the presence of $\mathrm{SH}$ electrons, but is well below the $\mathrm{SH}$ density $n_{\mathrm{SH}} \sim 30 \mathrm{~cm}^{-3}$. Figure 1(f) shows that most of the electrons have $E \lesssim 100 \mathrm{eV}$ and are from the $\mathrm{SH}$, but some MS electrons remain. This is consistent with the stagnation point being on the lower-density MS side of the $X$ line
$[19,20]$. At $E \sim 100 \mathrm{eV}$ the differential flux observed in the diffusion region varies quasiperiodically, due to the spacecraft spin and the electron distribution becoming anisotropic (discussed below). Figure 1(g) shows that the total plasma $\beta$ remains less than 1 in the diffusion region. The increase in $\beta$ across the diffusion region is due to the increase in $n$.

Figure 1(h) shows that the most intense electric field $\mathbf{E}$ activity occurs in the diffusion region. The electric field consists of a dc field $E \sim 10 \mathrm{mV} \mathrm{m}^{-1}$ in the $n$ direction, as well as a fluctuating field. The wavelet transform of the electric field $E_{x}$ in the spacecraft $x$ direction [Fig. 1(i)] shows that most of the power in the diffusion region has a broad frequency range, with most of the power near or below the local LH frequency $f_{\mathrm{LH}}$, consistent with waves produced by the LHDI [21]. Figure 1(j) plots $\left|E_{x}\right|$ over $\left|B_{y}\right|$ obtained by STAFF in the spacecraft $y$ direction. Near $f_{\mathrm{LH}}$, $E_{x} / B_{y}$ is much larger than the expected phase speed $v_{\perp}=$ $\omega_{\mathrm{LH}} / k_{\perp} \approx 1.3 \times 10^{6} \mathrm{~m} \mathrm{~s}^{-1}$ of electromagnetic LH waves with $k_{\perp}=\left(\rho_{e} \rho_{i}\right)^{-1 / 2}$ observed in simulations near the $X$ line [22], indicating that the dominant mode is electrostatic. Here $\rho_{e, i}$ are the electron and ion thermal gyroradii. Additionally, using the method from Ref. [21] to estimate the phase speed yields wave numbers $k_{\perp} \sim \rho_{e}^{-1}$, consistent with electrostatic LH waves. Therefore, we conclude that the electric fields observed in the diffusion region are primarily associated with electrostatic LH drift waves generated at the density gradient between the two plasmas.

We now investigate the electron distributions observed in the diffusion region. In Fig. 2 we plot the electron phasespace densities $f_{e}(E)$ versus $E$ when the diffusion region is observed. Because $\mathrm{C} 3$ and $\mathrm{C} 4$ are closely separated and at this time $\mathrm{C} 3$ and $\mathrm{C} 4$ 's spins are approximately $180^{\circ}$ out of phase, $f_{e}(E)$ at pitch angles $\theta=0^{\circ}$ and $180^{\circ}$ can be recorded almost simultaneously. Therefore, by combining $\mathrm{C} 3$ and $\mathrm{C} 4$ data we can observe $f_{e}(E)$ at all $\theta$ at twice the spacecraft spin period (once every $2 \mathrm{~s}$ ) in the diffusion region. The MS $f_{e}(E)$ is obtained at the beginning of Figs. 1(a)-(c) and the $\mathrm{SH} f_{e}(E)$ is obtained between 18:09:33 UT and 18:09:51 UT, when no MS electrons were observed in Fig. 1(c).

The development of electron heating parallel to $\mathbf{B}$ is seen in Fig. 2, where $f_{e}(E)$ is plotted for $\theta=0^{\circ}, 90^{\circ}$, and $180^{\circ}$. In Fig. 2(a) $f_{e}(E)$ is approximately equal for all $\theta$, meaning the plasma is isotropic. Figures 2(b) and 2(c) show $f_{e}(E)$ at $\theta=0^{\circ}$ and $180^{\circ}$ increasing above the distribution at $\theta=$ $90^{\circ}$ for $20 \mathrm{eV} \lesssim E \lesssim 1000 \mathrm{eV}$. The anisotropy in $f_{e}(E)$ is greatest in Fig. 2(c) and corresponds to the heating of SH electrons parallel to B. For $E>100 \mathrm{eV}, f_{e}(E)$ at $\theta=0^{\circ}$ and $180^{\circ}$ exceeds that of the $\mathrm{SH}$, indicating that the $\mathrm{SH}$ electrons reaching this point have been accelerated. In Figs. 2(d)-2(f) parallel heating is still observed but it becomes less pronounced as the density increases. Figure 3(b) shows a diagram of the spacecraft trajectory through the reconnection event based on the data in Figs. 1 

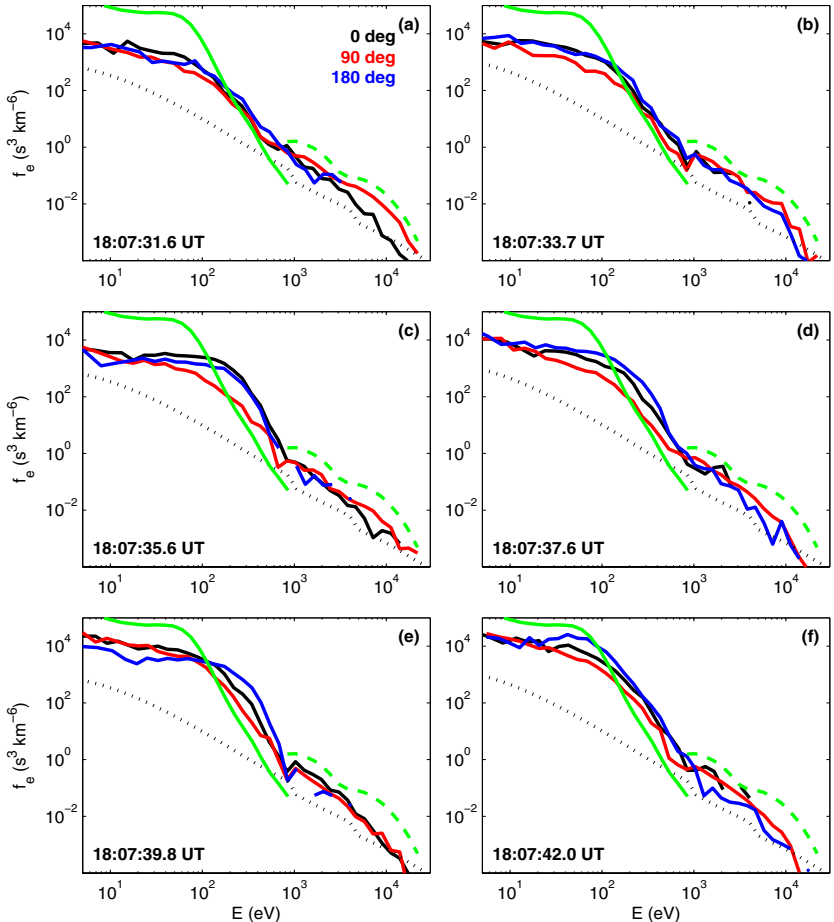

FIG. 2 (color online). Electron distributions obtained by combining $\mathrm{C} 3$ and $\mathrm{C} 4$ data in the diffusion region, indicated by the blue shading in Fig. 1. The distributions are obtained as $0.125 \mathrm{~s}$ energy sweeps, collected from HEEA and LEEA sensors on each spacecraft. Full pitch angle and energy coverage is achieved by combining data from $\mathrm{C} 3$ and $\mathrm{C} 4$ collected over a $1 \mathrm{~s}$ interval. The median time is stated in each panel. The green dashed and solid lines are the unperturbed MS $f_{e}(E)$ and $\mathrm{SH} f_{e}(E)$, respectively. Dotted lines indicate the instrumental one-count level.

and 2. The structure of $\mathbf{B}$ and $n$ is based on numerical simulations $[6,10]$.

Throughout the diffusion region MS electrons are observed. However, in each panel of Fig. $2 f_{e}(E)$ at $E \gtrsim 1000 \mathrm{eV}$ is below that of the unperturbed MS for all $\theta$. In the diffusion region $f_{e}(E)$ is nearly isotropic at MS energies, although $f_{e}(E)$ is typically largest at $\theta=90^{\circ}$. Since we do not observe strong asymmetries between $\theta=0^{\circ}$ and $\theta=180^{\circ}$ - which is expected for electron separatrix regions away from the diffusion region-the observations are consistent with $\mathrm{C} 3$ and $\mathrm{C} 4$ crossing the diffusion region.

From Fig. 2 it is evident that $f_{e}(E)$ is due to the mixing of MS and SH electrons. However, plasma mixing alone cannot explain the heating of SH electrons parallel to $\mathbf{B}$, similar to MS separatrix regions [23]. Additional processes must occur to heat the electrons above the $\mathrm{SH}$ temperature in the diffusion region. Possible mechanisms include electron trapping and acceleration and heating by $\mathrm{LH}$ waves.

The electron trapping model [8] argues that the electron distributions in the diffusion region are due to electron trapping and acceleration associated with the electric and magnetic field structure near the $X$ line. Electrons can be

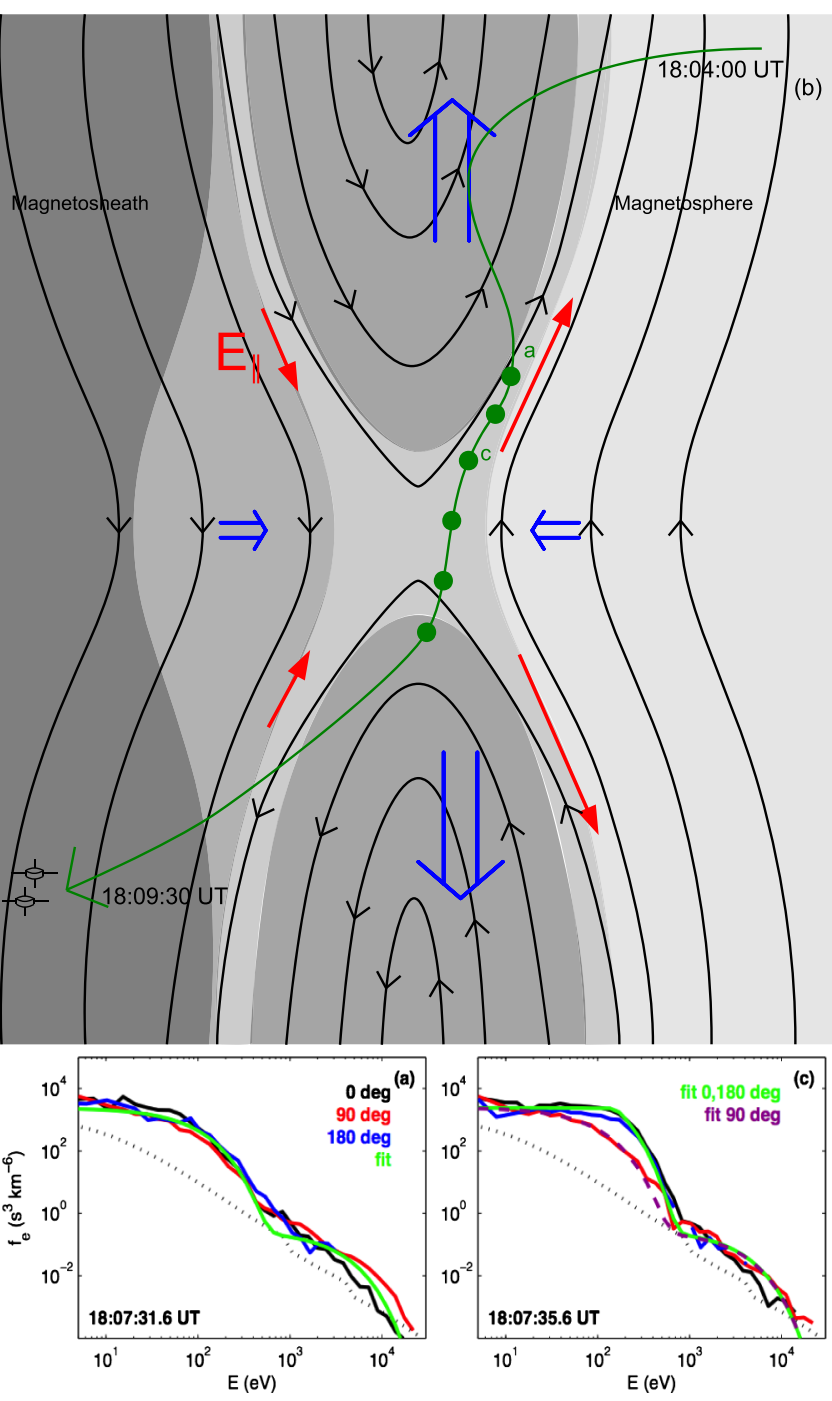

FIG. 3 (color online). Diagram of MP reconnection and $f_{e}(E)$ near the diffusion region. (b) Diagram of the spacecraft trajectory (green, gray line) through the reconnection event, showing $\mathbf{B}$ (black lines), ion flows (blue, gray double arrows), expected $\mathbf{E}_{\|}$ (red, gray arrows), and density (gray background, with darker gray corresponding to higher density). From top to bottom, the green, gray circles indicate the expected spacecraft positions at the times given in Figs. 2(a)-2(f). Panels (a) and (c) show $f_{e}(E)$ from Figs. 2(a) and 2(c) with fits to the data. In panel (a) the green curve is a two-Maxwellian fit to the data and in panel (c) the green solid and purple dashed curves, for $\theta=0^{\circ}, 180^{\circ}$ and $\theta=90^{\circ}$, respectively, are obtained using Liouville's theorem with $\Phi_{\|}=150 \mathrm{~V}$.

trapped due to a decrease in $B$ near the $X$ line and/or an accelerating potential due to a nonzero $\mathbf{E}_{\|}$. We observe the anisotropy in $f_{e}(E)$ on the MS side of the $X$ line where $\mathbf{B} \approx \mathbf{B}_{\mathrm{MS}}$ and $B_{\mathrm{MS}}$ is comparable to $B_{\mathrm{SH}}$, so the effect of magnetic trapping on $f_{e}(E)$ is negligible. Moreover, magnetic trapping cannot accelerate electrons at $\theta=0^{\circ}$ and $180^{\circ}$ to produce the observed heating in Fig. 2(c) [8]. Therefore, for electron trapping to explain the observed electron distributions a parallel potential $\Phi_{\|}$must be present. 
We now show that the observed $f_{e}(E)$ are consistent with electron trapping by a positive $\Phi_{\|}$. Figure 3(b) shows the spacecraft trajectory through the reconnection event and approximately where the distributions in Fig. 2 were observed. In Fig. 3(a) the $f_{e}(E)$ observed just outside the diffusion region near the ion outflow is well modeled as two isotropic Maxwellians with $T_{e}=2000 \mathrm{eV}$ and $60 \mathrm{eV}$ for the MS and SH populations. We obtain a fit to the diffusion region $f_{e}(E)$ [Fig. 3(c)] using Liouville's theorem for a positive $\Phi_{\|}$[8], where the fit in Fig. 3(a) is used as the ambient $f_{e}(E)$. Figure 3(c) shows $f_{e}(E)$ obtained for $\Phi_{\|}=150 \mathrm{~V}$. This fit reproduces all the features of the observed $f_{e}(E)$, namely, parallel heating for SH electrons, a flat distribution for $E \lesssim 100 \mathrm{eV}$, and an approximately isotropic MS population. On the SH side of the $X$ line we estimate $\Phi_{\|} \sim-100 \mathrm{~V}$, corresponding to $\mathbf{E}_{\|}$pointing toward the $X$ line, to reflect $\mathrm{SH}$ electrons and reduce $n_{\mathrm{SH}}$ near the $X$ line [10]. This situation is consistent with local trapping, where $\Phi_{\|}<0$ adjacent to the trapping region, making $f_{e}(E)$ in Fig. 3(a) the relevant ambient $f_{e}(E)$ for the fit [24]. We also estimate $\Phi_{\|}$using the asymptotic form $e \Phi_{\|} / k_{B} T_{e} \sim \pi \tilde{n}^{2} /\left(4 \tilde{B}^{2}\right) \sim 3$, [10] where $\tilde{n}$ and $\tilde{B}$ are the diffusion-region $n$ and $B$ normalized to the ambient $n$ and $B$ in Fig. 3(a). For $T_{e}=60 \mathrm{eV}$, we obtain $\Phi_{\|} \sim 180 \mathrm{~V}$, in agreement with the above estimate. In short, the observed $f_{e}(E)$ is consistent with $\mathbf{E}_{\|}$trapping and heating $\mathrm{SH}$ electrons but having little effect on the high-energy MS electrons.

LH waves provide a mechanism for heating electrons parallel to $\mathbf{B}$ and have been suggested as a possible contribution to anomalous resistivity [25,26] and diffusion across the MP [13]. The electric field associated with LH waves can resonantly accelerate electrons parallel to $\mathbf{B}$ [12]. Figure 1 shows that the electric field is primarily electrostatic and broadband, consistent with LH drift waves generated on the low- $\beta$ side of the density gradient $[21,27]$. For LH drift waves to effectively heat electrons, the electrons must remain in the diffusion region comparable to or longer than the typical damping time scale. This requires the inflow speed to be sufficiently slow that the electrons remain in the diffusion region long enough for significant heating to develop. Reference [12] showed that several ( 10) ion cyclotron periods are required, based on quasilinear simulations. We can estimate the time electrons are in the diffusion region by estimating the width of the diffusion region and the typical inflow speed. The typical width of the diffusion region is $d_{\mathrm{MS}} \sim c / \omega_{\mathrm{pi}(\mathrm{MS})} \sim 300 \mathrm{~km}$ for $n_{\mathrm{MS}}=0.5 \mathrm{~cm}^{-3}$, where $\omega_{\mathrm{pi}(\mathrm{MS})}$ is the MS ion plasma frequency. By assuming a nominal diffusion region length of $L \sim 10 d_{\text {MS }}$ we estimate an inflow speed of $\sim 25 \mathrm{~km} \mathrm{~s}^{-1}$ for both the MS and SH plasmas using the scalings of Ref. [19]. Therefore, we estimate the time the electrons are in the ion diffusion region to be $t \sim 12 \mathrm{~s} \sim 60 \Omega_{i}^{-1}$, where $\Omega_{i} \approx 5.3 \mathrm{~s}^{-1}$ is the ion cyclotron frequency in the diffusion region. This result indicates that both MS and SH electrons remain in the diffusion region long enough for $f_{e}(E)$ to be modified.

To heat the electrons the waves must resonate with the electrons in the diffusion region. This requires the $\mathrm{LH}$ phase speed to be comparable to the local electron thermal speed $v_{e}$. The resonant condition is [12]

$$
v_{\|}=\frac{\omega_{\mathrm{LH}}}{k_{\|}}=\sqrt{\frac{m_{i}}{m_{e}}}\left(\frac{\omega_{\mathrm{LH}}}{k_{\perp}}\right)^{1 / 2},
$$

where $v_{\|}$is the parallel phase speed, and $k_{\|}$and $k_{\perp}$ are the parallel and perpendicular wave numbers. For LH wave numbers $k_{\perp}=\rho_{e}^{-1}$ and $k_{\perp}=\left(\rho_{e} \rho_{i}\right)^{-1 / 2}$ we estimate $v_{\|} \sim$ $v_{e}$ and $v_{\|} \sim 10 v_{e}$, respectively. Therefore, LH drift waves with $k_{\perp} \rho_{e} \sim 1$, consistent with those in Fig. 1, can heat the local SH electrons parallel to $\mathbf{B}$. Electron trapping in the potential maxima of LH drift waves may also modify $f_{e}(E)$ if the amplitude is large enough. We estimate the potential maxima to be of order $\Phi \sim \rho_{e} E_{\max } \sim 10 \mathrm{~V}$. Normalizing to the local $T_{e}$ yields $e \Phi / k_{B} T_{e} \sim 0.1$, which agrees with previous observations [21], and is consistent with scattering of SH electrons, but inconsistent with trapping and the observed heating.

In conclusion, we have investigated the electron distributions in the ion diffusion region of an asymmetric reconnection near the subsolar point. The event shows large-scale evidence of reconnection, namely, ion outflows, a flow reversal, mixing of MS and $\mathrm{SH}$ electrons, parallel electron heating, and a magnetic field reversal.

The key results are as follows:

1. The diffusion region is observed on the MS side of the $X$ line at the density gradient. The region consists of a mixture of MS and SH electrons. However, only the SH electrons are heated parallel to $\mathbf{B}$.

2. The observed electron distributions are consistent with trapping of $\mathrm{SH}$ electrons by parallel electric fields. We estimate $\Phi_{\|}=150 \mathrm{~V}$, which traps SH electrons and produces the observed parallel heating but is too small to trap or heat MS electrons.

3. Lower hybrid drift waves are observed in the diffusion region, which satisfy the conditions required to heat $\mathrm{SH}$ electrons. However, since electron trapping alone explains the observed electron distributions, the lower hybrid waves likely do not contribute significantly to the observed electron heating.

The results presented in this Letter can be further studied and tested in the upcoming Magnetospheric Multiscale mission. The results suggest that the relative temperatures of the reconnecting plasmas are important and suggest that simulations should take into account the temperature difference when modeling MP reconnection.

We thank the ESA Cluster Active Archive team and the Cluster PI teams for data access and support. This work was supported by the Swedish National Space Board, Grant No. 128/11:2. 
[1] E. R. Priest and T. Forbes, Magnetic Reconnection: MHD Theory and Applications (Cambridge University Press, Cambridge, England, 2000).

[2] J. Birn et al., J. Geophys. Res. 106, 3715 (2001).

[3] M. Swisdak, B. N. Rogers, J. F. Drake, and M. A. Shay, J. Geophys. Res. 108, 1218 (2003).

[4] J. Birn, J. E. Borovsky, and M. Hesse, Phys. Plasmas 15, 032101 (2008).

[5] P. L. Pritchett, J. Geophys. Res. 113, A06210 (2008).

[6] K. G. Tanaka et al., Ann. Geophys. 26, 2471 (2008).

[7] L.-J. Chen et al., J. Geophys. Res. 113, A12213 (2008).

[8] J. Egedal, W. Fox, N. Katz, M. Porkolab, M. Oieroset, R. P. Lin, W. Daughton, and J. F. Drake, J. Geophys. Res. 113, A12207 (2008).

[9] X. Tang, C. Cattell, J. Dombeck, L. Dai, L. B. Wilson III, A. Breneman, and A. Hupach, Geophys. Res. Lett. 40, 2884 (2013).

[10] J. Egedal, A. Le, P. L. Pritchett, and W. Daughton, Phys. Plasmas 18, 102901 (2011).

[11] J. D. Scudder, R. D. Holdaway, W. S. Daughton, H. Karimabadi, V. Roytershteyn, C. T. Russell, and J. Y. Lopez, Phys. Rev. Lett. 108, 225005 (2012).

[12] I. H. Cairns and B. F. McMillan, Phys. Plasmas 12, 102110 (2005).

[13] A. Vaivads, M. André, S. C. Buchert, J.-E. Wahlund, A. N. Fazakerley, and N. Cornilleau-Wehrlin, Geophys. Res. Lett. 31, L03804 (2004).
[14] F. S. Mozer, M. Wilber, and J. F. Drake, Phys. Plasmas 18, 102902 (2011).

[15] P. L. Pritchett, F. S. Mozer, and M. Wilber, J. Geophys. Res. 117, A06212 (2012).

[16] P. L. Pritchett, Phys. Plasmas 20, 061204 (2013).

[17] C. P. Escoubet, M. Fehringer, and M. Goldstein, Ann. Geophys. 19, 1197 (2001).

[18] J.-H. Shue et al., J. Geophys. Res. 103, 17691 (1998).

[19] P. A. Cassak and M. A. Shay, Phys. Plasmas 14, 102114 (2007).

[20] K. Malakit, M. A. Shay, P. A. Cassak, and D. Ruffolo, Phys. Rev. Lett. 111, 135001 (2013).

[21] C. Norgren, A. Vaivads, Y. V. Khotyaintsev, and M. Andre, Phys. Rev. Lett. 109, 055001 (2012).

[22] V. Roytershteyn, W. Daughton, H. Karimabadi, and F. S. Mozer, Phys. Rev. Lett. 108, 185001 (2012).

[23] Y. V. Khotyaintsev, A. Vaivads, A. Retino, M. Andre, C. J. Owen, and H. Nilsson, Phys. Rev. Lett. 97, 205003 (2006).

[24] J. Egedal, A. Le, and W. Daughton, Phys. Plasmas 20, 061201 (2013).

[25] S. D. Bale, F. S. Mozer, and T. Phan, Geophys. Res. Lett. 29, 2180 (2002).

[26] I. Silin, J. Buchner, and A. Vaivads, Phys. Plasmas 12, 062902 (2005).

[27] N. A. Krall and P.C. Liewer, Phys. Rev. A 4, 2094 (1971). 